\title{
MULTISPHALERONS IN THE WEAK INTERACTIONS
}

\author{
Burkhard Kleihaus \\ Fachbereich Physik, Universität Oldenburg, Postfach 2503 \\ D-26111 Oldenburg, Germany \\ Jutta Kunz \\ Fachbereich Physik, Universität Oldenburg, Postfach 2503 \\ D-26111 Oldenburg, Germany \\ and \\ Instituut voor Theoretische Fysica, Rijksuniversiteit te Utrecht \\ NL-3508 TA Utrecht, The Netherlands
}

March 15, 1994

\begin{abstract}
We construct multisphaleron solutions in the weak interactions. The multisphaleron solutions carry Chern-Simons charge $n / 2$, where $n$ is an integer. The well-known sphaleron has $n=1$ and is spherically symmetric for vanishing mixing angle. In contrast the multisphalerons with $n>1$ are only axially symmetric, even for vanishing mixing angle. The greater $n$, the stronger the energy density deforms. While for small Higgs masses the energy of the multisphalerons is smaller than $n$ times the energy of the sphaleron, the energy of the multisphalerons is larger than $n$ times the energy of the sphaleron for large Higgs masses.
\end{abstract}

Univ. Utrecht-Preprint THU-94/04 


\section{Introduction}

In 1976 't Hooft [1] observed that the standard model does not absolutely conserve baryon and lepton number due to the Adler-Bell-Jackiw anomaly. In particular 't Hooft considered spontaneous fermion number violation due to instanton transitions between topologically inequivalent vacua. Fermion number violating tunnelling transitions at high energies might become observable at future accelerators $[2,3]$.

In 1983 Manton [4] considered the possibility of fermion number violation in the weak interactions from another point of view. He showed that there are non-contractible loops in configuration space and predicted the existence of a static, unstable solution of the field equations, a sphaleron [5], representing the top of the energy barrier between topologically distinct vacua.

It is this interpretation of the sphaleron solution, which makes it physically relevant. At finite temperature the energy barrier between distinct vacua can be overcome due to thermal fluctuations of the fields, and vacuum to vacuum transitions can occur, accompanied by a change of baryon and lepton number. The rate for baryon number violating processes is largely determined by a Boltzmann factor, containing the height of the barrier at a given temperature, and thus by the energy of the sphaleron [6-10].

In this letter we investigate the configuration space of the Weinberg-Salam theory further and construct multisphaleron solutions. In contrast to the sphaleron [5], which is spherically symmetric for vanishing mixing angle, the multisphaleron solutions are only axially symmetric, even for vanishing mixing angle. The appropriate ansatz for the multisphalerons represents a generalization of the axially symmetric ansatz for the sphaleron at finite mixing angle [11,12], preserving the invariance under parity.

Like the multiinstantons the multisphalerons can be labelled by an integer $n$, which is associated with the topological charge of these solutions. This integer $n$ appears in the ansatz for the multisphalerons, representing a winding number with respect to the azimuthal angle $\phi$. While $\phi$ covers the full trigonometric circle once, the fields wind $n$ times around. The ansatz is thus analogous to the ansatz of Manton [13] and Rebbi and Rossi [14] for multimonopole solutions of an $\mathrm{SU}(2)$ gauge theory with a Higgs triplet.

From the ansatz the energy functional and the equations of motion for the fields are obtained. Considering only vanishing mixing angle, the resulting system of coupled non-linear partial differential equations is solved numerically. For $n=1$ spherical symmetry and the well known sphaleron [5] are recovered.

In section 2 we briefly review the Weinberg-Salam lagrangian, discuss the ansatz and present the resulting energy functional. In section 3 we exhibit the multisphaleron solutions with $n \leq 5$. We present our conclusions in section 4 . 


\section{Weinberg-Salam Lagrangian}

Let us consider the bosonic sector of the Weinberg-Salam theory

$$
\mathcal{L}=-\frac{1}{4} F_{\mu \nu}^{a} F^{\mu \nu, a}-\frac{1}{4} f_{\mu \nu} f^{\mu \nu}+\left(D_{\mu} \Phi\right)^{\dagger}\left(D^{\mu} \Phi\right)-\lambda\left(\Phi^{\dagger} \Phi-\frac{v^{2}}{2}\right)^{2}
$$

with the $\mathrm{SU}(2)_{\mathrm{L}}$ field strength tensor

$$
F_{\mu \nu}^{a}=\partial_{\mu} V_{\nu}^{a}-\partial_{\nu} V_{\mu}^{a}+g \epsilon^{a b c} V_{\mu}^{b} V_{\nu}^{c},
$$

with the $\mathrm{U}(1)$ field strength tensor

$$
f_{\mu \nu}=\partial_{\mu} A_{\nu}-\partial_{\nu} A_{\mu}
$$

and the covariant derivative for the Higgs field

$$
D_{\mu} \Phi=\left(\partial_{\mu}-\frac{i}{2} g \tau^{a} V_{\mu}^{a}-\frac{i}{2} g^{\prime} A_{\mu}\right) \Phi
$$

The gauge symmetry is spontaneously broken due to the non-vanishing vacuum expectation value $v$ of the Higgs field

$$
\langle\Phi\rangle=\frac{v}{\sqrt{2}}\left(\begin{array}{l}
0 \\
1
\end{array}\right),
$$

leading to the boson masses

$$
M_{W}=\frac{1}{2} g v, \quad M_{Z}=\frac{1}{2} \sqrt{\left(g^{2}+g^{\prime 2}\right)} v, \quad M_{H}=v \sqrt{2 \lambda} .
$$

The mixing angle $\theta_{w}$ is determined by the relation $\tan \theta_{w}=g^{\prime} / g$, and the electric charge is $e=g \sin \theta_{w}$.

$\mathrm{SU}(2)$ gauge field configurations can be classified by a charge, the Chern-Simons charge. The Chern-Simons current

$$
K_{\mu}=\frac{g^{2}}{16 \pi^{2}} \varepsilon_{\mu \nu \rho \sigma} \operatorname{Tr}\left(\mathcal{F}^{\nu \rho} \mathcal{V}^{\sigma}+\frac{2}{3} i g \mathcal{V}^{\nu} \mathcal{V}^{\rho} \mathcal{V}^{\sigma}\right)
$$

$\left(\mathcal{F}_{\nu \rho}=1 / 2 \tau^{i} F_{\nu \rho}^{i}, \mathcal{V}_{\sigma}=1 / 2 \tau^{i} V_{\sigma}^{i}\right)$ is not conserved, its divergence $\partial^{\mu} K_{\mu}$ represents the $\mathrm{SU}(2)$ part of the $\mathrm{U}(1)$ anomaly of the baryon current. The Chern-Simons charge of a configuration is given by

$$
N_{\mathrm{CS}}=\int d^{3} r K^{0}
$$

For the vacua the Chern-Simons charge is identical to the integer winding number, while the sphaleron has a Chern-Simons charge of $1 / 2$ [5]. 
Let us now consider the ansatz for the multisphaleron solutions. Following Manton[13] and Rebbi and Rossi [14] we define a set of orthonormal vectors

$$
\begin{aligned}
& \vec{u}_{1}^{(n)}(\phi)=(\cos n \phi, \sin n \phi, 0), \\
& \vec{u}_{2}^{(n)}(\phi)=(0,0,1) \\
& \vec{u}_{3}^{(n)}(\phi)=(\sin n \phi,-\cos n \phi, 0)
\end{aligned}
$$

and expand the fields as follows

$$
\begin{gathered}
V_{i}^{a}(\vec{r})=u_{j}^{i(1)}(\phi) u_{k}^{a(n)}(\phi) w_{j}^{k}(\rho, z), \\
A_{i}(\vec{r})=u_{j}^{i(1)}(\phi) a_{j}(\rho, z), \\
\Phi(\vec{r})=i \tau^{i} u_{j}^{i(n)}(\phi) h_{j}(\rho, z) \frac{v}{\sqrt{2}}\left(\begin{array}{l}
0 \\
1
\end{array}\right) .
\end{gathered}
$$

Invariance under rotations about the $z$-axis and under parity leads to the conditions $[11,12]$

$$
\begin{gathered}
w_{1}^{1}(\rho, z)=w_{2}^{1}(\rho, z)=w_{1}^{2}(\rho, z)=w_{2}^{2}(\rho, z)=w_{3}^{3}(\rho, z)=0 \\
a_{1}(\rho, z)=a_{2}(\rho, z)=0 \\
h_{3}(\rho, z)=0 .
\end{gathered}
$$

The resulting axially symmetric energy functional $E$

$$
E=\frac{1}{2} \int\left(E_{w}+E_{a}+v^{2} E_{h}\right) d \phi \rho d \rho d z
$$

then has the contributions

$$
\begin{gathered}
E_{w}=\left(\partial_{\rho} w_{3}^{1}+\frac{1}{\rho}\left(n w_{1}^{3}+w_{3}^{1}\right)-g w_{1}^{3} w_{3}^{2}\right)^{2}+\left(\partial_{z} w_{3}^{1}+\frac{n}{\rho} w_{2}^{3}-g w_{2}^{3} w_{3}^{2}\right)^{2} \\
+\left(\partial_{\rho} w_{3}^{2}+\frac{1}{\rho} w_{3}^{2}+g w_{1}^{3} w_{3}^{1}\right)^{2}+\left(\partial_{z} w_{3}^{2}+g w_{2}^{3} w_{3}^{1}\right)^{2}+\left(\partial_{\rho} w_{2}^{3}-\partial_{z} w_{1}^{3}\right)^{2} \\
E_{a}=\left(\partial_{\rho} a_{3}+\frac{1}{\rho} a_{3}\right)^{2}+\left(\partial_{z} a_{3}\right)^{2} \\
E_{h}=\left(\partial_{\rho} h_{1}-\frac{g}{2} w_{1}^{3} h_{2}\right)^{2}+\left(\partial_{z} h_{1}-\frac{g}{2} w_{2}^{3} h_{2}\right)^{2}+\left(\partial_{\rho} h_{2}+\frac{g}{2} w_{1}^{3} h_{1}\right)^{2}+\left(\partial_{z} h_{2}+\frac{g}{2} w_{2}^{3} h_{1}\right)^{2} \\
+\left(\frac{n}{\rho} h_{1}+\frac{g}{2}\left(w_{3}^{1} h_{2}-w_{3}^{2} h_{1}\right)-\frac{g^{\prime}}{2} a_{3} h_{1}\right)^{2}+\left(\frac{g}{2}\left(w_{3}^{1} h_{1}+w_{3}^{2} h_{2}\right)-\frac{g^{\prime}}{2} a_{3} h_{2}\right)^{2} \\
+\frac{\lambda v^{2}}{2}\left(h_{1}^{2}+h_{2}^{2}-1\right)^{2} .
\end{gathered}
$$


It is still invariant under gauge transformations generated by

$$
U=e^{i \Gamma(\rho, z) \tau^{i} u_{3}^{i(n)}}
$$

analogous to [11-14]. Note, that under this transformation the 2-D Higgs doublets $\left(h_{1}, h_{2}\right)$ and $\left(w_{3}^{1}, w_{3}^{2}-n / g \rho\right)$ transform with angle $\Gamma(\rho, z)$ and $2 \Gamma(\rho, z)$, respectively, while the $2-\mathrm{D}$ gauge field $\left(w_{1}^{3}, w_{2}^{3}\right)$ transforms inhomogeneously. Here we fix this gauge degree of freedom by choosing the gauge condition $[11,12]$

$$
\partial_{\rho} w_{1}^{3}+\partial_{z} w_{2}^{3}=0
$$

Changing to spherical coordinates and extracting the trivial $\theta$-dependence we specify the ansatz further $[11,12]$

$$
\begin{gathered}
w_{1}^{3}(r, \theta)=\frac{2}{g r} F_{1}(r, \theta) \cos \theta, \quad w_{2}^{3}(r, \theta)=-\frac{2}{g r} F_{2}(r, \theta) \sin \theta \\
w_{3}^{1}(r, \theta)=-\frac{2 n}{g r} F_{3}(r, \theta) \cos \theta, \quad w_{3}^{2}(r, \theta)=\frac{2 n}{g r} F_{4}(r, \theta) \sin \theta \\
a_{3}(r, \theta)=\frac{2}{g^{\prime} r} F_{7}(r, \theta) \sin \theta \\
h_{1}(r, \theta)=F_{5}(r, \theta) \sin \theta, \quad h_{2}(r, \theta)=F_{6}(r, \theta) \cos \theta .
\end{gathered}
$$

Note, that the spherically symmetric ansatz for the sphaleron corresponds to $n=1$ and $F_{1}(r, \theta)=F_{2}(r, \theta)=F_{3}(r, \theta)=F_{4}(r, \theta)=f(r), F_{5}(r, \theta)=F_{6}(r, \theta)=h(r)$, and $F_{7}(r, \theta)=0$ (where the functions $f(r)$ and $h(r)$ correspond to those of ref. [5]).

The above ansatz, when inserted into the classical equations of motion in the chosen gauge, yields a set of coupled partial differential equations for the functions $F_{i}(r, \theta)$, to be solved numerically subject to certain boundary conditions. To obtain regular, finite energy solutions with the imposed symmetries, we take as boundary conditions for the functions $F_{i}(r, \theta)$

$$
\begin{array}{rlll}
r=0 & : & \left.F_{i}(r, \theta)\right|_{r=0}=0, & i=1, \ldots, 7, \\
r \rightarrow \infty & : & \left.F_{i}(r, \theta)\right|_{r=\infty}=1, & i=1, \ldots, 6,\left.\quad F_{7}(r, \theta)\right|_{r=\infty}=0 \\
\theta=0: & \left.\partial_{\theta} F_{i}(r, \theta)\right|_{\theta=0}=0, & i=1, \ldots, 7 \\
\theta=\pi / 2 & : & \left.\partial_{\theta} F_{i}(r, \theta)\right|_{\theta=\pi / 2}=0, & i=1, \ldots, 7 .
\end{array}
$$

The Chern-Simons charge of the multisphaleron solutions can be evaluated analogously to the Chern-Simons charge of the sphaleron [5]. At spatial infinity the vector fields are pure gauge configurations

$$
\tau^{a} V_{i}^{a}=-\frac{2 i}{g} \partial_{i} U U^{\dagger}
$$


with

$$
U(\vec{r})=\exp \left(i \Omega(r, \theta)\left(\sin \theta \tau^{i} u_{1}^{i(n)}+\cos \theta \tau^{i} u_{2}^{i(n)}\right)\right) .
$$

The proper gauge for evaluating the Chern-Simons charge is the gauge with $U=1$ at infinity, which is obtained with the transformation $U^{\dagger}$ and $\Omega(0)=0$ and $\Omega(\infty)=\pi / 2$. The Chern-Simons charge of the multisphaleron solutions is then given by

$$
N_{\mathrm{CS}}=\frac{1}{2 \pi^{2}} \int d^{3} r Q(\vec{r})
$$

where the Chern-Simons density is determined by $[15,16]$

$$
Q(\vec{r})=n \frac{\sin ^{2} \Omega}{r^{2}} \frac{\partial \Omega}{\partial r}+\text { derivative terms } .
$$

Since the derivative terms do not contribute (because of the boundary conditions) the Chern-Simons charge of the multisphaleron solutions is given by

$$
N_{\mathrm{CS}}=n / 2 .
$$

The Chern-Simons charge of the multisphalerons corresponds to their baryonic charge, $Q_{B}=n / 2$, since the $\mathrm{U}(1)$ field does not contribute to their baryon number [5].

\section{Multisphaleron solutions}

We here restrict the numerical calculations to the limit of vanishing mixing angle. In this limit the $\mathrm{U}(1)$ field decouples and the function $F_{7}(r, \theta)$ can consistently be set to zero. The case of non-vanishing mixing angle will be considered later [15]. Subject to the boundary conditions (25) we then solve the equations numerically, using the dimensionless coordinate $x=g v r$. We fix the parameters $g=0.65$ and $M_{W}=80 \mathrm{GeV}$. The Higgs mass is varied.

The numerical calculations are based on the Newton-Raphson method. The equations are discretized on a non-equidistant grid in $x$ and an equidistant grid in $\theta$, where typical grids used have sizes $50 \times 20$ and $100 \times 20$ covering integration regions $0<x<60$ up to $0<x<180$ and $0<\theta<\pi / 2$. The numerical error for the functions is estimated to be on the order of $10^{-3}$.

To obtain the multisphaleron solutions we start with the $n=1$ sphaleron solution as initial guess and increase the value of $n$ slowly. This numerical procedure thus involves the construction of intermediate configurations, where $n$ takes on non-integer values. At each integer values of $n$ a multisphaleron solution is reached. 
In the following we exhibit the axially symmetric multisphaleron solutions. In particular we show as gauge invariant quantities, the energy density $\varepsilon$, defined by

$$
E=\frac{1}{4 \pi} \int \varepsilon(\vec{x}) x^{2} d x \sin \theta d \theta d \phi
$$

where $E$ is the energy in $\mathrm{TeV}$, and the magnitude of the Higgs field $\Phi$ in units of $v / \sqrt{2}$. In Figs. 1a and 1b we show the energy density $\varepsilon$ of the multisphalerons with $n=2$ and $n=5$ for the Higgs mass $M_{H}=M_{W}$, and in Figs. 2a and $2 \mathrm{~b}$ we show the corresponding magnitude of the Higgs field $\Phi$. The energy density is strongly peaked along the $\rho$-axis, with the maximum shifting outward with increasing $n$. The magnitude of the Higgs field, being zero at the origin, remains small along the $\rho$-axis for increasingly longer intervals with increasing $n$. This is also demonstrated in the following comparison of the sphaleron and all multisphalerons up to $n=5$. In Figs. $3 \mathrm{a}$ and $3 \mathrm{~b}$ we show the energy density along the $\rho$-axis and along the $z$-axis for the multisphalerons up to $n=5$ for $M_{H}=M_{W}$, and in Figs. $4 \mathrm{a}$ and $4 \mathrm{~b}$ we show the corresponding magnitude of the Higgs field.

The energy $E(n)$ of the multisphalerons from $n=2$ to $n=5$ is shown in Table 1 for the Higgs masses $M_{H}=M_{W} / 10, M_{H}=M_{W}$ and $M_{H}=10 M_{W}$ and compared with the corresponding energy $E(1)$ of the sphaleron, by considering the ratio $E(n) / n E(1)$. While for small Higgs masses the energy of the multisphalerons is smaller than $n$ times the energy of the sphaleron, for large Higgs masses the energy of the multisphalerons is larger than $n$ times the energy of the sphaleron.

\section{Conclusions}

We have constructed multisphaleron solutions in the weak interactions for vanishing mixing angle. The extension to finite mixing angle is in progress [15].

While the sphaleron is spherically symmetric for vanishing mixing angle, the multisphaleron solutions are only axially symmetric. They can be characterized by an integer $n$, related to the winding of the fields in the azimuthal angle $\phi$. With increasing $n$ the energy density of the multisphalerons becomes increasingly deformed, the maximum occurring along the $\rho$-axis at increasing distance from the origin.

The energy of the multisphalerons is on the order of $n$ times the sphaleron energy. For small Higgs masses the energy of the multisphalerons is less than $n$ times the energy of the sphaleron, $E(n)<n E(1)$, while for large Higgs masses the energy of the multisphalerons is greater than $n$ times the energy of the sphaleron, $E(n)>n E(1)$.

While the sphaleron has Chern-Simons charge $N_{\mathrm{CS}}=1 / 2$, the multisphaleron solutions have Chern-Simons charge $N_{\mathrm{CS}}=n / 2$. We conjecture, that symmetric non-

contractible loops can be constructed, leading from a vacuum with Chern-Simons charge 
$N_{\mathrm{CS}}=0$ to a topologically distinct vacuum with Chern-Simons charge $N_{\mathrm{CS}}=n$, passing the multisphaleron solution with $N_{\mathrm{CS}}=n / 2$ midway.

At finite temperature such paths involving multisphalerons should allow for thermal fermion number violating transitions, corresponding to tunnelling transitions via multiinstantons at zero temperature. Since multiinstantons with winding number $n$ possess $n$ fermion zero modes, we expect to encounter $n$ fermion zero modes along vacuum to vacuum paths, passing the $n$-th multisphaleron.

Acknowledgement

We gratefully acknowledge discussions with Yves Brihaye. 


\section{References}

[1] G. 't Hooft, Symmetry breaking through Bell-Jackiw Anomalies, Phys. Rev. Lett. 37 (1976) 8.

[2] A. Ringwald, High energy breakdown of perturbation theory in the electroweak instanton sector, Nucl. Phys. B330 (1990) 1.

[3] M. Mattis, and E. Mottola, eds., "Baryon Number Violation at the SSC?", World Scientific, Singapore (1990).

[4] N. S. Manton, Topology in the Weinberg-Salam theory, Phys. Rev. D28 (1983) 2019.

[5] F. R. Klinkhamer, and N. S. Manton, A saddle-point solution in the WeinbergSalam theory, Phys. Rev. D30 (1984) 2212.

[6] V. A. Kuzmin, V. A. Rubakov, and M. E. Shaposhnikov, On anomalous electroweak baryon-number non-conservation in the early universe, Phys. Lett. B155 (1985) 36.

[7] P. Arnold, and L. McLerran, Sphalerons, small fluctuations, and baryon-number violation in electroweak theory, Phys. Rev. D36 (1987) 581.

[8] P. Arnold, and L. McLerran, The sphaleron strikes back: A response to objections to the sphaleron approximation, Phys. Rev. D37 (1988) 1020.

[9] L. Carson, X. Li, L. McLerran, and R.-T. Wang, Exact computation of the smallfluctuation determinant around a sphaleron, Phys. Rev. D42 (1990) 2127.

[10] E. W. Kolb, and M. S. Turner, "The Early Universe", Addison-Wesley Publishing Company, Redwood City (1990).

[11] B. Kleihaus, J. Kunz, and Y. Brihaye, The electroweak sphaleron at physical mixing angle, Phys. Lett. B273 (1991) 100.

[12] J. Kunz, B. Kleihaus, and Y. Brihaye, Sphalerons at finite mixing angle, Phys. Rev. D46 (1992) 3587.

[13] N. S. Manton, Complex structure of monopoles, Nucl. Phys. B135 (1978) 319.

[14] C. Rebbi and P. Rossi, Multimonopole solutions in the Prasad-Sommerfield limit, Phys. Rev. D22 (1980) 2010.

[15] B. Kleihaus and J. Kunz, in preparation

[16] Y. Brihaye and J. Kunz, in preparation 


\section{Table}

Table 1

\begin{tabular}{||c|c|c|c||}
\multicolumn{4}{c}{$E(n) / \mathrm{TeV}(E(n) / n E(1))$} \\
\hline \hline$n$ & $M_{H}=M_{W} / 10$ & $M_{H}=M_{W}$ & $M_{H}=10 M_{W}$ \\
\hline 1 & $7.468(1.000)$ & $8.665(1.000)$ & $11.375(1.000)$ \\
2 & $13.791(0.923)$ & $17.140(0.989)$ & $24.937(1.096)$ \\
3 & $19.881(0.887)$ & $25.879(0.996)$ & $40.030(1.173)$ \\
4 & $25.908(0.867)$ & $34.931(1.008)$ & $56.298(1.237)$ \\
5 & $31.931(0.855)$ & $44.288(1.022)$ & $73.521(1.293)$ \\
\hline \hline
\end{tabular}

\section{$6 \quad$ Figure captions}

\section{Figure 1:}

The energy density $\varepsilon$ (in $\mathrm{TeV}$ ) is shown as a function of the dimensionless coordinates $\rho$ and $z$ for the multisphalerons with $n=2$ (1a) and $n=5(1 \mathrm{~b})$.

Figure 2:

The magnitude of the Higgs field $\Phi$ (in units of $v / \sqrt{2}$ ) is shown as a function of the dimensionless coordinates $\rho$ and $z$ for the multisphalerons with $n=2(2 \mathrm{a})$ and $n=5$ (2b).

\section{Figure 3:}

The energy density $\varepsilon$ (in TeV) along the $\rho$-axis (3a) and $z$-axis (3b) is shown as a function of the dimensionless coordinate $\rho$ resp. $z$ for the sphaleron with $n=1$ and the multisphalerons from $n=2$ to $n=5$.

Figure 4:

The magnitude of the Higgs field $\Phi$ (in units of $v / \sqrt{2}$ ) along the $\rho$-axis (3a) and $z$-axis $(3 \mathrm{~b})$ is shown as a function of the dimensionless coordinate $\rho$ resp. $z$ for the sphaleron with $n=1$ and the multisphalerons from $n=2$ to $n=5$. 
This figure "fig1-1.png" is available in "png" format from: http://arxiv.org/ps/hep-ph/9403289v1 
Fig. 1a

$$
\varepsilon(\rho, \mathrm{z}) \mathrm{n}=2
$$

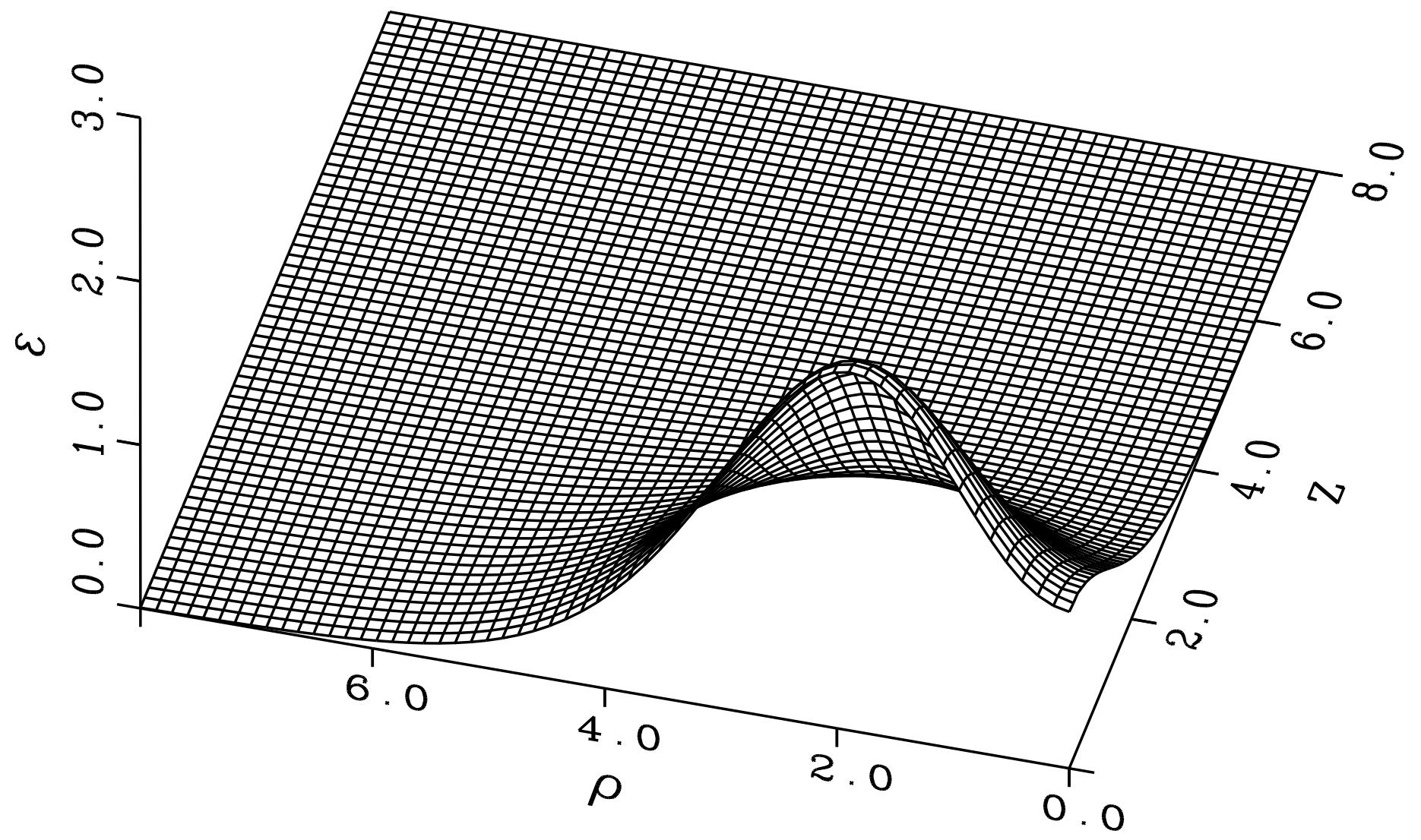


Fig. 1b

$$
\varepsilon(\rho, \mathrm{z}) \mathrm{n}=5
$$

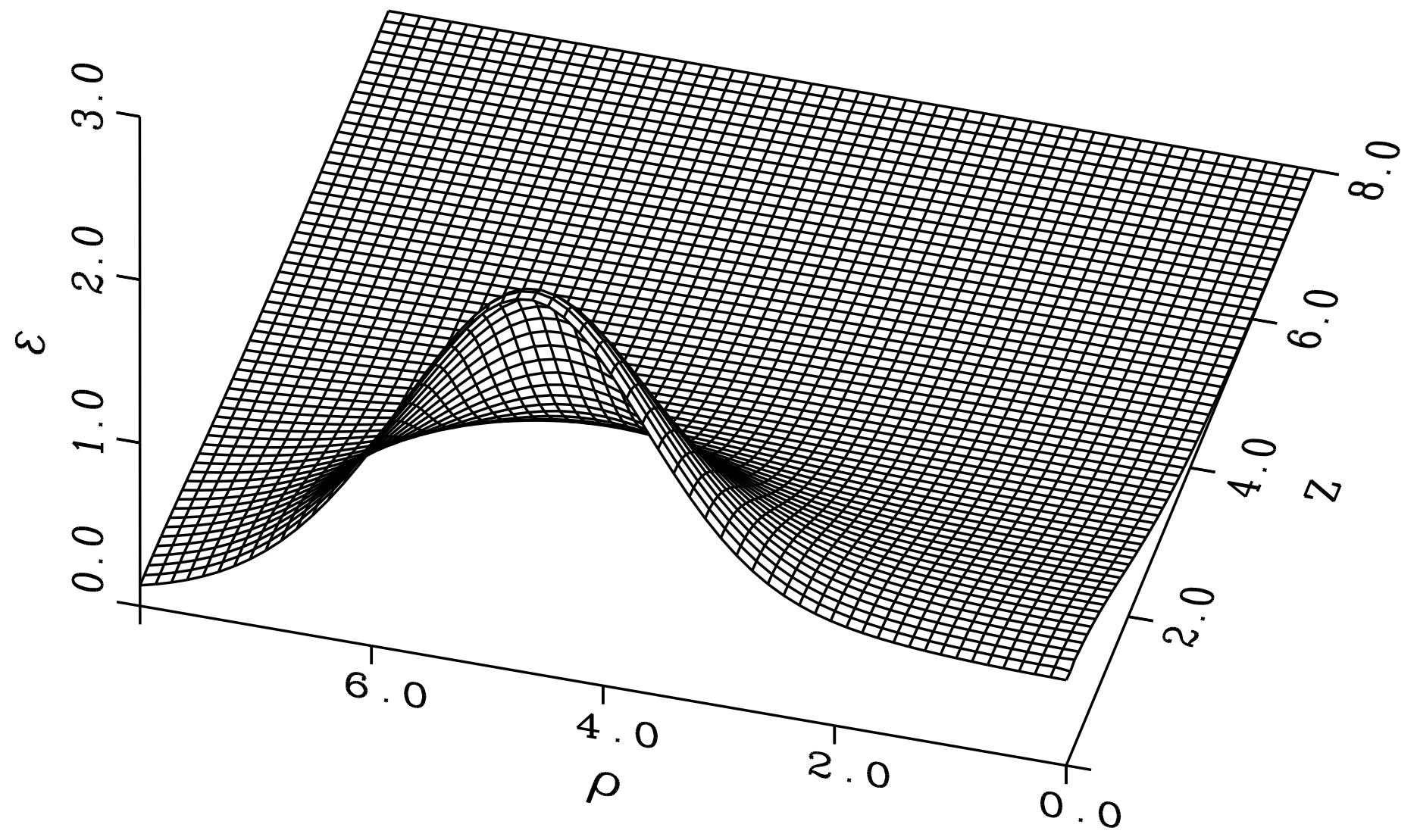


This figure "fig2-1.png" is available in "png" format from: http://arxiv.org/ps/hep-ph/9403289v1 
This figure "fig1-2.png" is available in "png" format from: http://arxiv.org/ps/hep-ph/9403289v1 
This figure "fig2-2.png" is available in "png" format from: http://arxiv.org/ps/hep-ph/9403289v1 


\section{Fig. 2a}

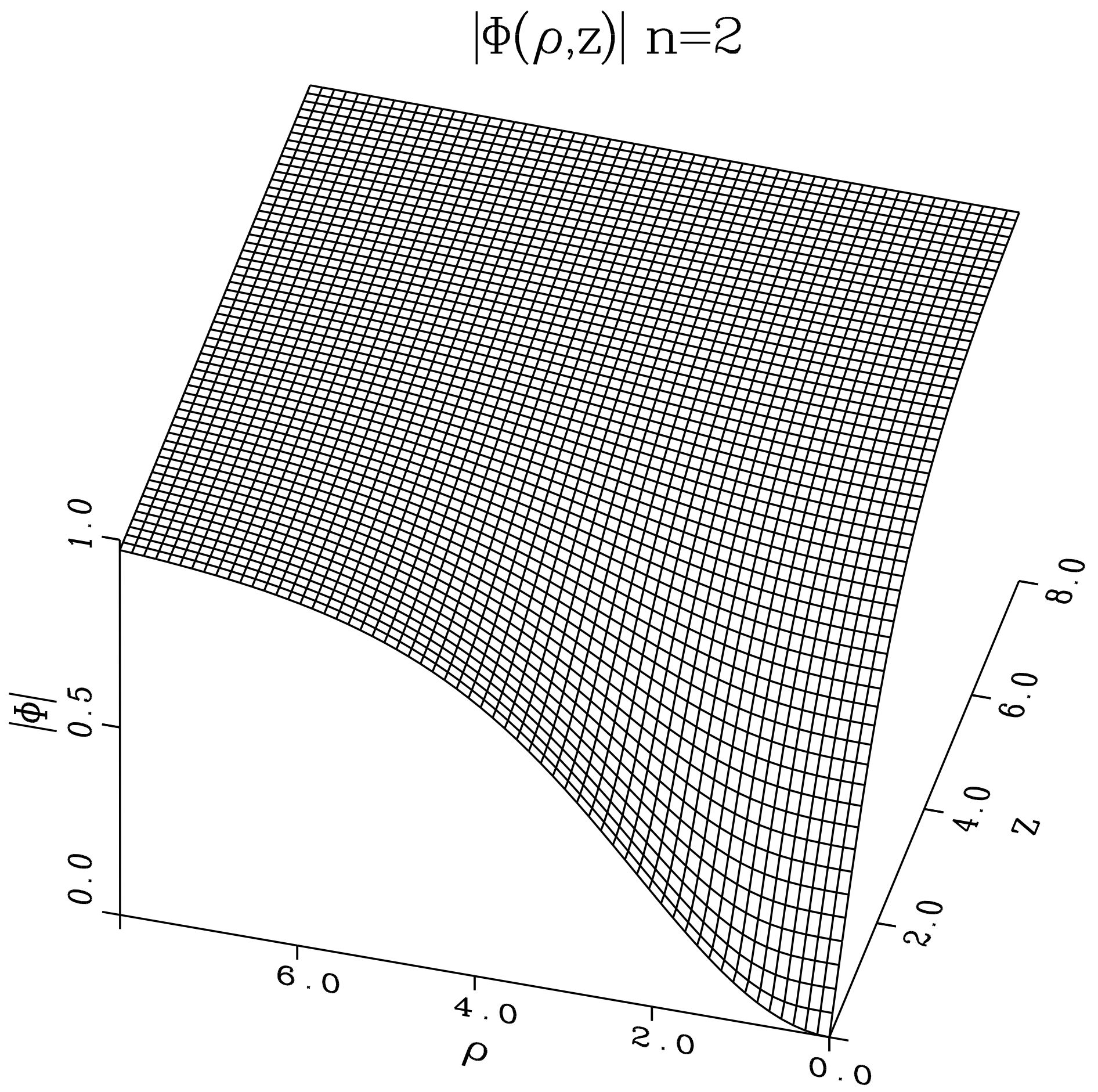




\section{Fig. 2b}

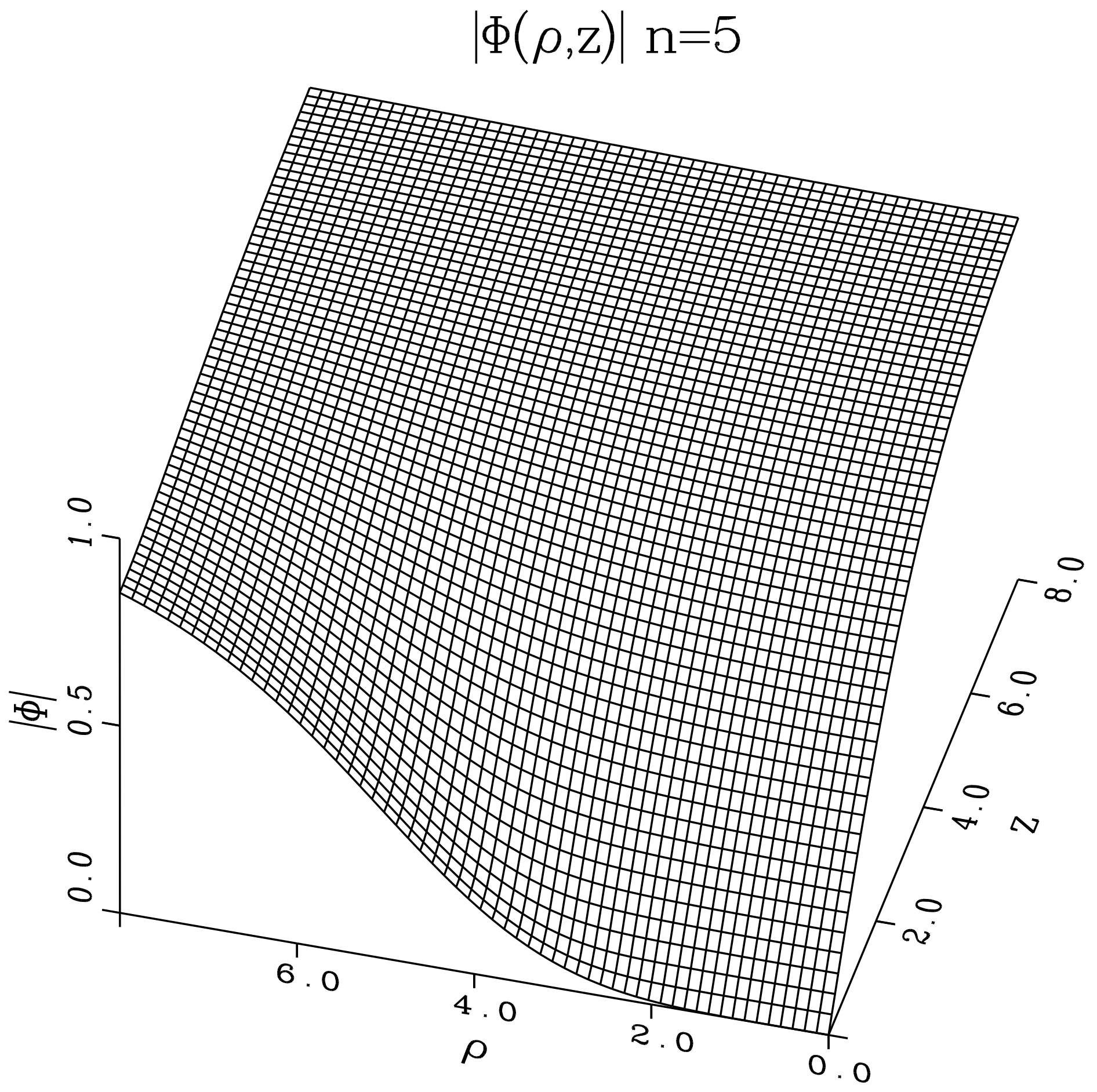


This figure "fig1-3.png" is available in "png" format from: http://arxiv.org/ps/hep-ph/9403289v1 
This figure "fig2-3.png" is available in "png" format from: http://arxiv.org/ps/hep-ph/9403289v1 
Fig. 3a

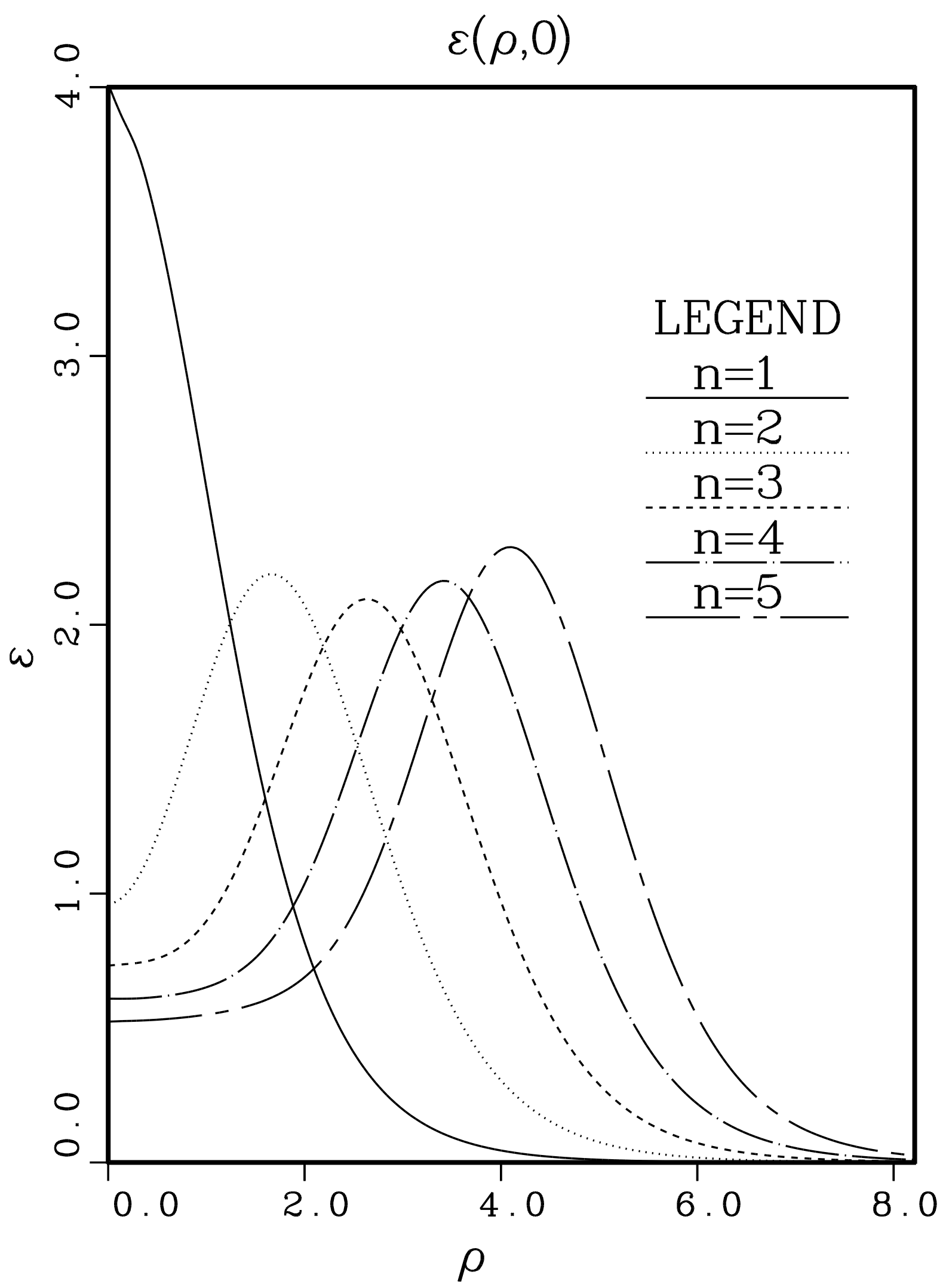


Fig. 3b

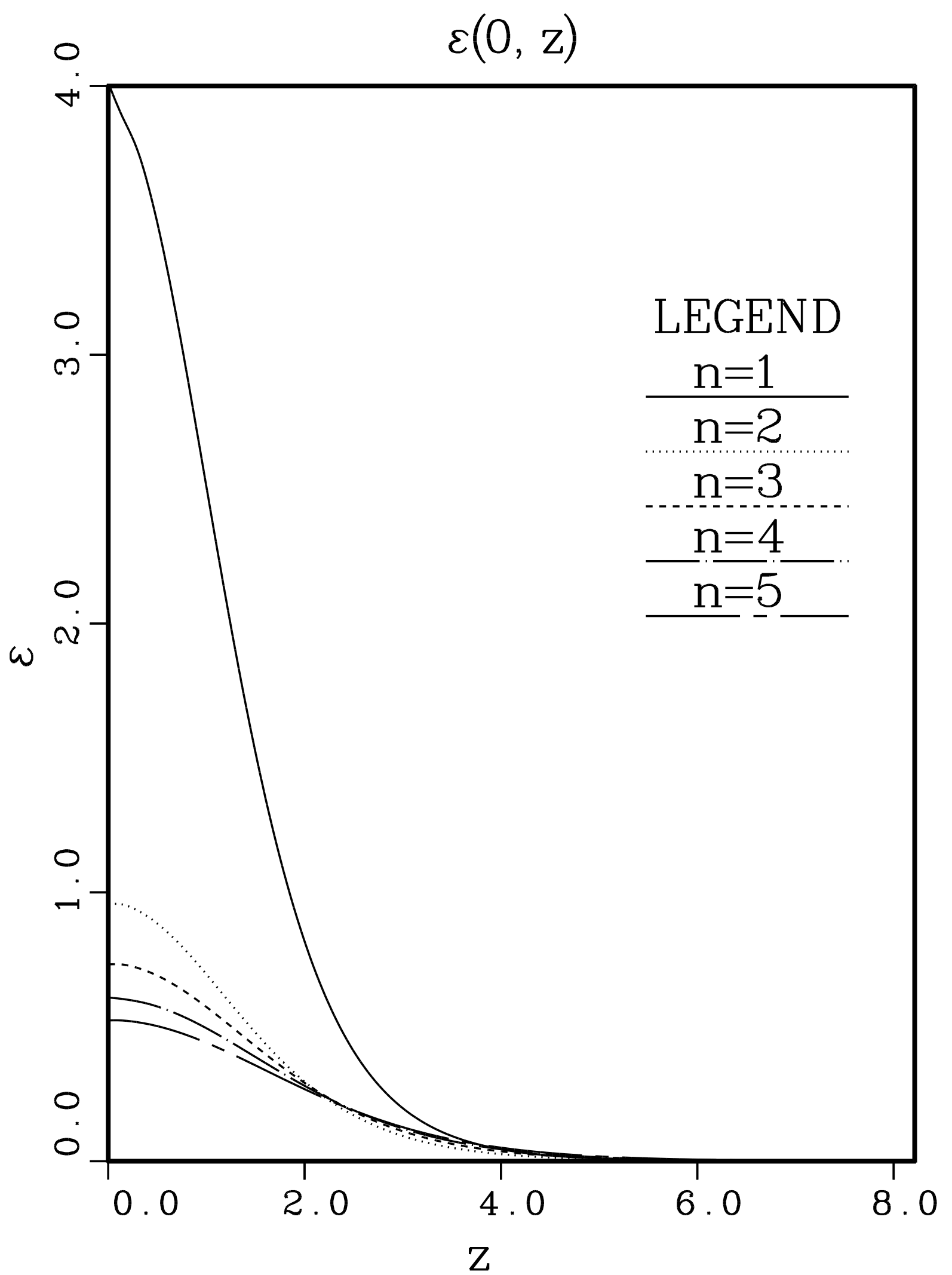


This figure "fig1-4.png" is available in "png" format from: http://arxiv.org/ps/hep-ph/9403289v1 
This figure "fig2-4.png" is available in "png" format from: http://arxiv.org/ps/hep-ph/9403289v1 
Fig. $4 a$

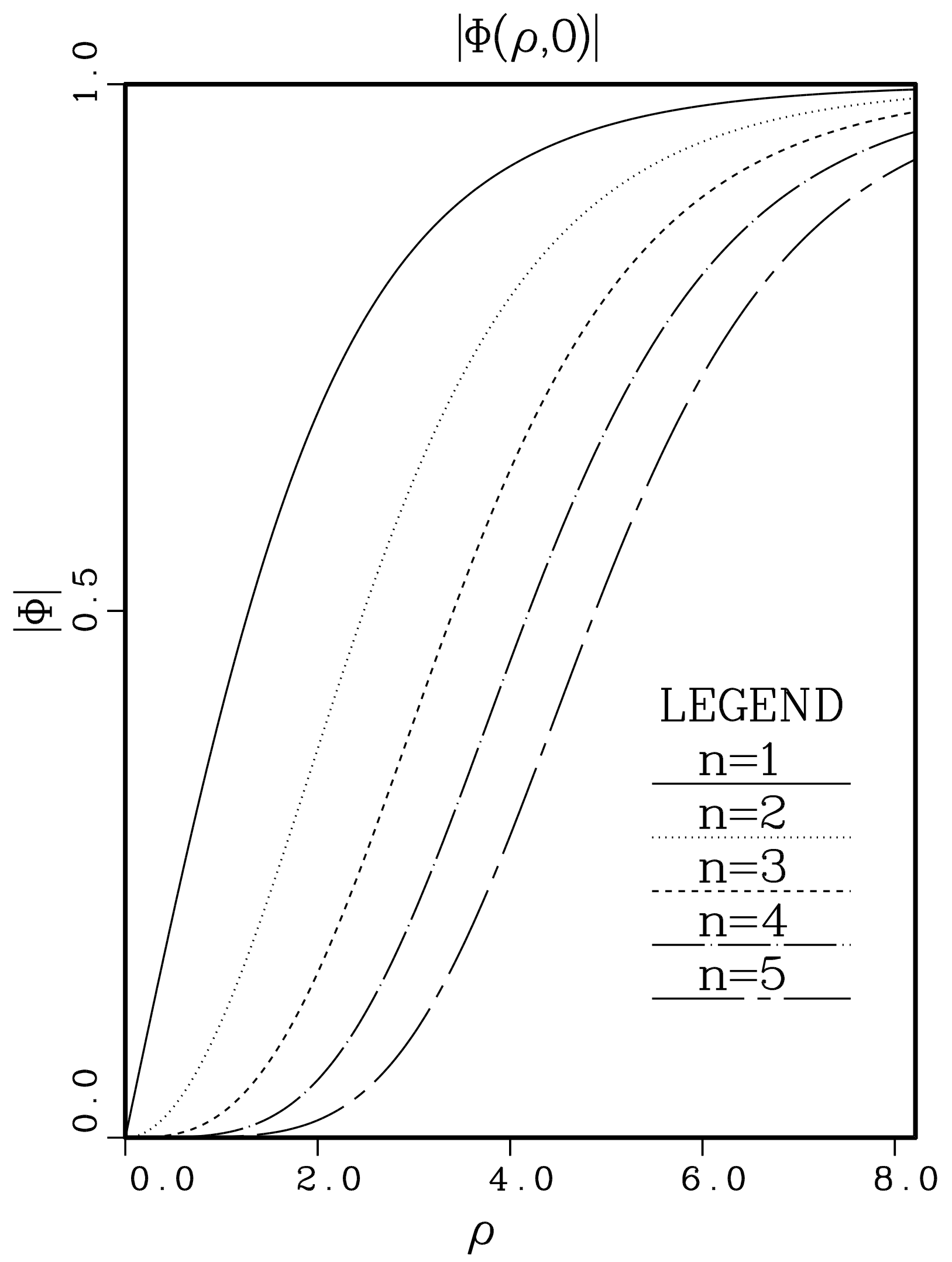




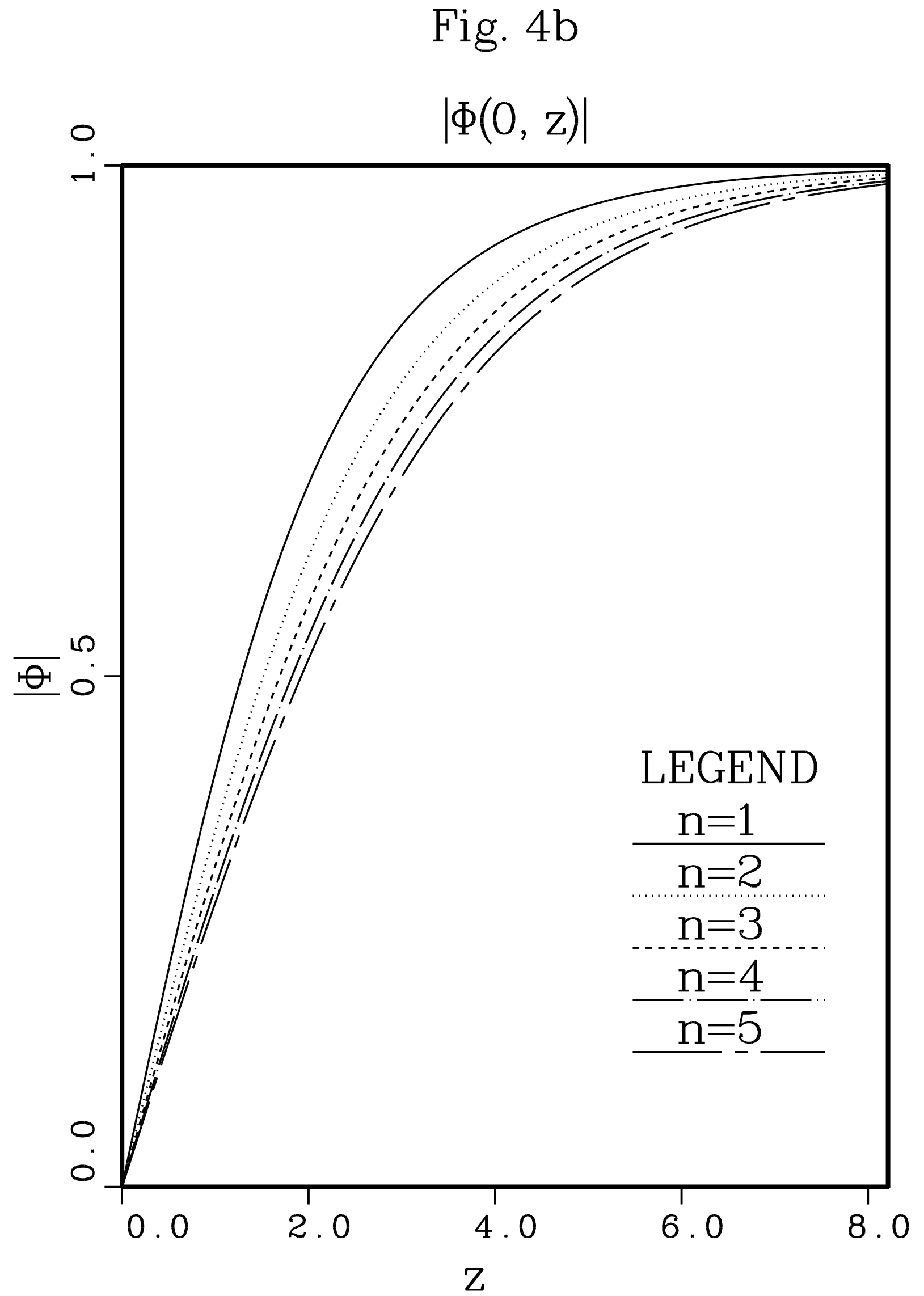

